\title{
POSICIONAMENTO DE EXTRATORES DE CÁPSULA POROSA EM SOLO ARENOSO NA CITRICULTURA FERTIRRIGADA POR GOTEJAMENTO
}

\section{LEANDRO CAIXETA SALOMÃO ${ }^{1}$; THAIS REGINA DE SOUZA ${ }^{2}$; ROBERTO LYRA VILLAS BÔAS ${ }^{3}$; THOMAS FIORI DE ANDRADE ${ }^{4}$; LETÍCIA CECÍLIA FORATTO $^{5}$ e ALESSANDRO J. M. SANTOS}

1 Doutorando do Curso de Pós-Graduação em Irrigação e Drenagem, da Faculdade de Ciências Agronômicas FCA/UNESP. Rua José Barbosa de Barros 1780, CEP: 18610-307, Botucatu SP, e-mail: lsalomao@fca.unesp.br

2 Pós-Doutoranda do Instituto Agronômico, Centro de Solos e Recursos Ambientais, Campinas SP, e-mail: tr_souza@yahoo.com.br

3 Professor Doutor do Departamento de Recursos Naturais/Ciência do Solo, FCA/UNESP, e-mail: rlvboas@fca.unesp.br

4 Doutorando do Curso de Pós-Graduação em Irrigação e Drenagem, FCA/UNESP, e-mail: tfandrade@fca.unesp.br

5 Doutoranda do Curso de Pós-Graduação em Irrigação e Drenagem, FCA/UNESP, e-mail: lc_foratto@yahoo.com.br

${ }^{6}$ Doutorando do Curso de Pós-Graduação em Energia na Agricultura, FCA/UNESP, e-mail: amarques@fca.unesp.br

\section{RESUMO}

A eficiência do monitoramento da fertirrigação com o auxilio de extratores de cápsulas porosas está diretamente relacionada ao seu correto posicionamento, visto que os nutrientes aplicados via água de irrigação apresentam mobilidade diferente ao longo do perfil do solo. Dessa forma, o objetivo deste trabalho foi avaliar a distribuição de $\mathrm{NO}_{3}, \mathrm{P}$ e K na solução do solo, quando aplicados via fertirrigação pelo sistema de irrigação localizada por gotejamento na citricultura, visando definir o adequado posicionamento dos extratores de solução em solo arenoso. Para aferição da concentração dos íons na solução do solo, foram instalados extratores de solução do solo em quatro diferentes distâncias $(5,15,25$, e $35 \mathrm{~cm})$ e profundidades $(15,30,60$ e $90 \mathrm{~cm})$ com relação a um emissor localizado sob a projeção da copa das plantas. $\mathrm{O}$ experimento foi conduzido em pomar de laranjeira Valência sobre o porta-enxerto citrumelo Swingle, na cidade de Reginópolis/SP. O delineamento experimental empregado foi o de blocos ao acaso, o arranjo dos tratamentos constituiu um fatorial 4 x 4 com cinco repetições. Foram instalados 16 extratores de solução do solo por bloco, totalizando 80 extratores no experimento. De acordo com os resultados obtidos recomenda-se instalar um extrator de solução para determinar $\mathrm{P}$ e $\mathrm{K}$, a $15 \mathrm{~cm}$ de distância do emissor na horizontal e a $30 \mathrm{~cm}$ de profundidade; e para $\mathrm{NO}_{3}{ }^{-}$outro extrator localizado a $25 \mathrm{~cm}$ de distância do emissor na horizontal e a $30 \mathrm{~cm}$ de profundidade. O extrator de solução mostrou ser uma ferramenta sensível, capaz de determinar a mobilidade dos íons no bulbo molhado.

Palavras-chave: irrigação, solução do solo, nutrientes.

\author{
SALOMÃO, L. C.; SOUZA, T. R. de; BÔAS, R. L. V.; ANDRADE, T. F. de; \\ FORATTO, L. C.; SANTOS, A. J. M. \\ POSITION OF POROUS CAPSULE EXTRACTORS FOR DRIP FERTIGATED \\ CITRUS IN SANDY SOIL
}




\section{ABSTRACT}

The efficiency of monitoring fertigation by means of porous capsule extractors is directly related to its correct placement, since the nutrients applied through fertigation have different mobility throughout the soil profile. The objective of this study was to evaluate the distributions of $\mathrm{NO}_{3}, \mathrm{P}$, and $\mathrm{K}$ in the soil solution, when applied in citrus in sandy soil through drip fertigation systems, aiming to define the proper placement of the solution extractors. In order to measure the concentration of ions in the soil solution, the extractors were installed at four different distances $(5,15,25$, and $35 \mathrm{~cm})$ and depths $(15,30,60$, and $90 \mathrm{~cm})$ with an emitter located under the projection of the tree canopy. The experiment was conducted in a Valencia orange orchard on citrumelo Swingle rootstock, in Reginópolis/SP. The experimental design was randomized blocks and the treatment arrangement was a $4 \times 4$ factorial design with five repetitions. Sixteen soil solution extractors were installed per block, with a total of 80 extractors in the experiment. According to the obtained results, to determine $\mathrm{P}$ and $\mathrm{K}$, it is recommended to install the solution extractor at $15 \mathrm{~cm}$ horizontal and $30 \mathrm{~cm}$ depth from the emitter. For $\mathrm{NO}_{3}$, the extractor recommended placement is $25 \mathrm{~cm}$ horizontal and $30 \mathrm{~cm}$ depth. The soil solution extractor proved to be a sensitive tool, capable of determining the ions mobility in the wet bulb.

Keywords: irrigation, soil solution, nutrients.

\section{INTRODUÇÃO}

O Brasil vem ganhando espaço na citricultura mundial, tornando-se líder na produção de laranja e de suco concentrado. A região sudeste, sobretudo o estado de São Paulo, é referência na produção de citros. Aproximadamente $21 \%$ das áreas neste estado são irrigadas, o que corresponde aproximadamente 125 mil hectares (Coelho, 2010). Grande parte dessa área emprega o sistema de irrigação localizada, associado à técnica de fertirrigação, que permite a aplicação de nutrientes em menores quantidades e com maior frequência, nesta condição o manejo da fertirrigação utilizando extratores de solução do solo auxilia no monitoramento da adubação.

Um ponto de fundamental importância na tomada de decisão para instalação dos extratores de solução é o estudo da movimentação dos íons no solo, visto que os nutrientes aplicados ao solo apresentam movimentação diferenciada ao longo do perfil. A mobilidade dos íons no solo é dependente de vários fatores. Para Sousa (2006), o movimento de íons no solo está relacionado com a intensidade de percolação da água e com o comportamento de cada um em relação às condições de fixação, adsorção e lixiviação, que é função de cada íon e do tipo de solo em que se encontra.

A lixiviação dos íons através do perfil do solo, segundo Reichardt (1990), é uma das principais causas de perdas de nutrientes, contribuindo sensivelmente para a acidificação do solo. Isto indica a necessidade de adotar manejo de água e nutrientes com critério. Solos arenosos são comuns na citricultura brasileira e o manejo da fertirrigação nestes solos é complexo, pois normalmente apresentam baixa capacidade de retenção de água e nutrientes.

$\mathrm{O}$ nutriente de maior mobilidade no solo é o nitrogênio na forma de nitrato $\left(\mathrm{NO}_{3}\right)$. Segundo Villas Bôas et al. (1999) o nitrato move-se para a periferia da frente de molhamento e a concentração desse íon logo abaixo do emissor é pequena. Embora as plantas absorvam prontamente nitrato, amônio e uréia, respostas para $\mathrm{NO}_{3}$ são normalmente mais rápidas, 
porque o nitrato é carregado pela água até a superfície da raiz via fluxo de massa. Portanto, se o manejo da irrigação for inadequado, por exemplo, excesso de água, o nitrato será lixiviado abaixo da zona radicular.

A taxa de fixação de fósforo no solo é alta e, consequentemente, o seu movimento é limitado, com isto, várias fontes e métodos de aplicação de fósforo vêm sendo testados para evitar, pelo menos parcialmente, os problemas de distribuição desse elemento no solo. Embora o movimento do fósforo dependa de muitos fatores químicos e físicos do solo; a textura, a taxa de aplicação e a quantidade de água aplicada, são as variáveis que mais afetam seu movimento (Villas Bôas et al., 2002).

A movimentação de potássio no solo aplicado via água de irrigação por gotejamento, foi objeto de estudo de Zanini (1991), que observou maior concentração de potássio no centro do bulbo, cerca de 30 a $40 \mathrm{~cm}$ de profundidade, sendo possível determinar aumento da concentração de potássio até $80 \mathrm{~cm}$ de profundidade logo após a fertirrigação. $\mathrm{O}$ autor relata que os locais de maiores concentrações do íon coincidem com os locais de maiores valores de umidade, evidenciando seu caminhamento por fluxo de massa.

A junção de três fatores; umidade do solo, concentração de nutrientes e presença de raízes, pode levar a uma melhor localização dos extratores. Deve-se considerar que a presença de água e nutrientes também pode induzir o aumento de raízes, favorecendo sua absorção. Portanto, se as condições do solo forem adequadas para o desenvolvimento radicular à concentração de nutrientes e a água poderão indicar o melhor posicionamento dos extratores no solo.

Segundo Blanco (2006) a dimensão do bulbo molhado na irrigação por gotejamento é muito variável, pois depende do tipo de solo, da umidade do solo no momento inicial da aplicação de água, da vazão dos gotejadores, dentre outros fatores e isso difículta a padronização dos extratores. Portanto, o objetivo deste trabalho foi avaliar a distribuição de $\mathrm{NO}_{3}$, P e K na solução do solo, quando aplicados via fertirrigação pelo sistema de irrigação localizada por gotejamento na citricultura, visando definir o adequado posicionamento dos extratores de solução em solo arenoso.

\section{MATERIAL E MÉTODOS}

O experimento foi conduzido no período de setembro e outubro de 2008 na fazenda Emu, pertencente à empresa Citrovita. Localizada na cidade de Reginópolis, estado de São Paulo, à latitude $21^{\circ} 49^{\prime} 45^{\prime \prime}$ sul, longitude $49^{\circ} 08^{\prime} 27^{\prime \prime}$ oeste e altitude $460 \mathrm{~m}$. O talhão escolhido para a instalação do experimento foi o da variedade de laranjeira Valência sobre o porta-enxerto citrumelo Swingle, plantadas no ano de 2003, em espaçamento de $7 \mathrm{~m}$ entre linhas e $4 \mathrm{~m}$ entre plantas.

Para a caracterização química (Raij et al., 2001) e física (Camargo 1986) do solo foram retiradas amostras nas camadas de $0-20 \mathrm{~cm}, 20-40 \mathrm{~cm}$ e $40-60 \mathrm{~cm}$ de profundidade (Tabelas 1 e 2), também foram feitas avaliações físicas na camada de 60-80 cm. 
Tabela 1. Caracterização química do solo na área experimental

\begin{tabular}{|c|c|c|c|c|c|c|c|c|c|c|}
\hline Camadas & $\mathbf{p H}$ & M.O. & $\mathbf{P}_{\text {resina }}$ & $\mathbf{H}+\mathbf{A l}$ & $\mathbf{K}$ & $\mathbf{C a}$ & Mg & SB & CTC & $\mathbf{V}$ \\
\hline $\mathbf{c m}$ & $\mathrm{CaCl}_{2}$ & $\mathrm{~g} \mathrm{dm}^{-3}$ & $\mathrm{mg} \mathrm{dm}^{-3}$ & \multicolumn{6}{|c|}{$\mathrm{mmol}_{\mathrm{c}} \mathrm{dm}^{-3}$} & $\%$ \\
\hline $0-20$ & 5,0 & 9 & 57 & 21 & 3,0 & 14 & 5 & 22 & 44 & 51 \\
\hline $20-40$ & 5,0 & 8 & 58 & 21 & 2,9 & 17 & 5 & 25 & 45 & 54 \\
\hline $40-60$ & 4,9 & 7 & 26 & 20 & 3,1 & 15 & 5 & 23 & 43 & 53 \\
\hline
\end{tabular}

M.O. = matéria orgânica, $\mathrm{SB}=$ soma de bases, $\mathrm{CTC}=$ capacidade de troca de cátions, $\mathrm{V}=$ saturação por bases.

Tabela 2. Caracterização física do solo na área experimental

\begin{tabular}{|c|c|c|c|c|c|}
\hline Camadas & $\mathrm{CC}$ & $\mathbf{P M}$ & Areia & Argila & Silte \\
\hline $\mathbf{c m}$ & \multicolumn{2}{|c|}{$\mathrm{cm} \mathrm{cm}^{-3}$} & \multicolumn{3}{|c|}{$\mathrm{g} \mathrm{kg}^{-1}$} \\
\hline $0-20$ & 0,102 & 0,050 & 819 & 131 & 51 \\
\hline $20-40$ & 0,126 & 0,070 & 827 & 125 & 49 \\
\hline $40-60$ & 0,160 & 0,090 & 802 & 146 & 53 \\
\hline $60-80$ & 0,170 & 0,110 & 746 & 206 & 48 \\
\hline
\end{tabular}

$\mathrm{CC}=$ capacidade de campo, $\mathrm{PM}=$ ponto de murcha permanente.

A quantidade de adubo aplicado foi definida com base no histórico de análises de solo e folha e também em função da produtividade esperada do talhão e correspondeu a 130, 25 e $120 \mathrm{~kg} \mathrm{ha}^{-1}$, respectivamente de $\mathrm{N}, \mathrm{P}_{2} \mathrm{O}_{5}$ e $\mathrm{K}_{2} \mathrm{O}$ (Quaggio et al., 2005), nas formas de nitrato de amônio, ácido fosfórico e cloreto de potássio branco. No período de avaliação foram aplicadas $25 \%$ da dose recomendada de $\mathrm{N}$ e $\mathrm{K}_{2} \mathrm{O}$ e $80 \%$ da dose recomendada de $\mathrm{P}_{2} \mathrm{O}_{5}$. A injeção dos fertilizantes foi realizada com o injetor tipo venturi, duas vezes por semana.

Utilizou-se o sistema de irrigação localizada por gotejamento, onde cada linha de plantio recebia uma linha lateral de irrigação, com emissores autocompensantes, espaçados entre si a 0,6 m, formando uma faixa molhada contínua, com largura máxima de $0,8 \mathrm{~m}$, os emissores forneciam uma vazão de $2,3 \mathrm{~L} \mathrm{~h}^{-1}$ e trabalharam com pressão de serviço de 20 mca.

O manejo da irrigação foi realizado com o auxílio de três baterias tensiômetros de punção instalados a $15 \mathrm{~cm}$ de distância do emissor e nas profundidades de 30 e $60 \mathrm{~cm}$, sendo a profundidade efetiva do sistema radicular da cultura de $60 \mathrm{~cm}$ (Pires et al., 2005). Adotou-se um turno de rega de dois dias, obteve-se uma lâmina média de $3,5 \mathrm{~mm}$. A curva característica de retenção da água no solo foi determinada conforme o método descrito por Camargo (1986) e ajustada pelo modelo proposto por Van Genuchten (1980), para as profundidades de 0-20, $20-40,40-60$ e $60-80 \mathrm{~cm}$.

O delineamento experimental empregado foi o de blocos ao acaso, o arranjo dos tratamentos constituiu um fatorial 4 x 4 com cinco repetições, compreendendo quatro distâncias do emissor 5, 15, 25 e $35 \mathrm{~cm}$, e quatro profundidades 15, 30, 60 e $90 \mathrm{~cm}$ (Figura 1). 


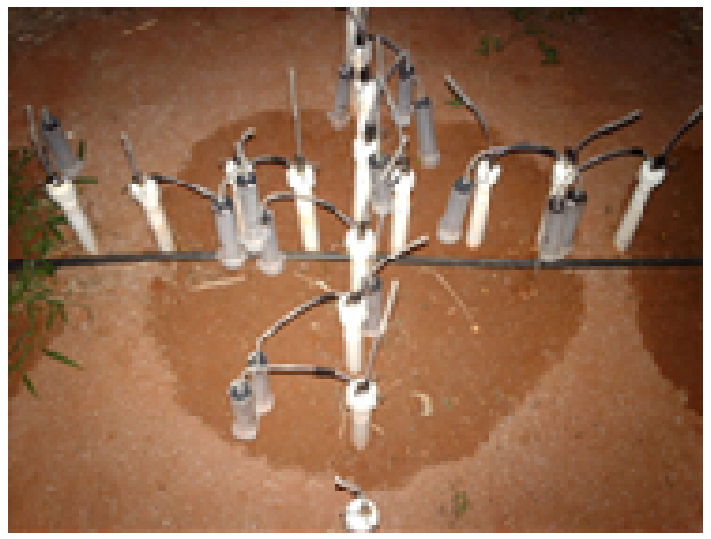

Figura 1. Bloco de extratores de solução do solo no experimento.

O extrator de solução do solo era composto de tubo de PVC, conectado na extremidade inferior a uma cápsula de cerâmica porosa. Na extremidade superior o extrator era vedado com rolha, e por esta passavam dois tubos de naylon, em um deles se aplicava o vácuo no outro ficava conectado uma seringa por onde se coletava a solução. Após a fertirrigação era aplicado nos extratores vácuo de $70 \mathrm{kPa}$, com o auxílio de uma bomba de vácuo elétrica, e após este procedimento era feita à coleta da solução. Foram realizadas nove coletas de solução do solo ao longo do experimento (uma coleta por semana).

No momento da realização da coleta da solução, era verificada a umidade do solo por meio de tensiômetros de punção, foram instaladas duas baterias de tesiômetros nas mesmas posições que foram instalados os extratores de solução. Para auxiliar na compreensão da movimentação dos íons no perfil do solo valores de $\mathrm{pH}$ e condutividade elétrica (CE) também foram avaliados.

As determinações de $\mathrm{pH}, \mathrm{CE}, \mathrm{NO}_{3}$ e $\mathrm{K}$ na solução do solo foram realizadas no próprio campo, sem filtragem ou digestão, logo após sua coleta. Para determinação dos valores de $\mathrm{pH}$, foi utilizado o pHmetro portátil, PG 1400, da marca Gehaka. Os valores de CE foram determinados utilizando o condutivímetro portátil, Twin Cond, da marca Horiba. Medidores de íons específicos da marca Horiba (Cardy) foram utilizados para determinar $\mathrm{NO}_{3}$ e $\mathrm{K}$ na solução do solo. Todos os equipamentos foram calibrados de acordo com a recomendação dos fabricantes.

Uma parte da solução extraída nas diferentes posições foi acondicionada em frascos de plástico devidamente identificados e encaminhados ao laboratório para análise de fósforo de acordo com o método descrito por Raij et al. (2001).

Os valores de $\mathrm{CE}, \mathrm{NO}_{3}, \mathrm{P}$ e $\mathrm{K}$ medidos na solução foram corrigidos para a umidade correspondente a capacidade de campo, equivalente a tensão de $10 \mathrm{kPa}$. Os resultados foram submetidos à análise de variância (ANOVA - Teste F a 5\% e 1\% de probabilidade) e ao teste de comparação de médias (Tukey a $5 \%$ de probabilidade), utilizando o programa estatístico SISVAR (Ferreira, 2003).

\section{RESULTADOS E DISCUSSÃO}

\subsection{Valores de umidade, pH e condutividade elétrica na solução do solo}

A umidade do solo foi maior próxima do emissor, independente da profundidade, chegando a $34 \%$ na profundidade de $15 \mathrm{~cm}$ e a $5 \mathrm{~cm}$ de distância horizontal do emissor. Como 
o solo da área experimental era arenoso o bulbo formado ficou menor na superfície e mais longo em profundidade, isso pode ser observado pela destruição da umidade no solo (Tabela 3). Chama atenção o valor de $30 \%$ de umidade a $90 \mathrm{~cm}$ de profundidade e a $5 \mathrm{~cm}$ de distância do emissor; nesta profundidade o solo apresenta acréscimo de argila (Tabela 2).

$\mathrm{Na}$ irrigação por gotejo, a água aplicada pelo gotejador penetra no solo e move-se para baixo e para os lados formando um bulbo. O tamanho e a forma do bulbo são afetados principalmente pela vazão do gotejador, tipo de solo e tempo de aplicação, portanto, a distribuição da água no solo na irrigação por gotejo depende dos atributos físico-hidráulicos do solo (Laurindo, 2005).

Os valores de $\mathrm{pH}$ na solução do solo de maneira geral foram baixos, próximos de 4,2, valores maiores foram observados na superfície (15 e $30 \mathrm{~cm}$ de profundidade) e distantes do emissor (25 e $35 \mathrm{~cm}$ de distância horizontal). As fontes de nitrogênio e fósforo aplicadas, respectivamente, nitrato de amônio e ácido fosfórico, possuem caráter ácido, e isso pode ter causado maior acidificação próxima do local de emissão dos fertilizantes (Tabela 4). Condições de acidez elevada são desfavoráveis ao desenvolvimento da maioria das culturas. Segundo Malavolta (2006) variações nos valores de $\mathrm{pH}$ afetam direta ou indiretamente a maioria dos nutrientes e sua disponibilidade para as plantas.

Maiores valores de condutividade elétrica ocorreram a $60 \mathrm{~cm}$ de profundidade e distantes do emissor, 25 e $35 \mathrm{~cm}$ na horizontal (1,3 dS m-1). Próximo do emissor, $5 \mathrm{~cm}$ de distância horizontal e $15 \mathrm{~cm}$ de profundidade, foram observados baixos valores (Tabela 5). $\mathrm{O}$ valor limiar de condutividade elétrica para a cultura do citros é de 1,4 dS m-1 (Maas, 1993), no período amostrado sempre foram observados valores inferiores ao limiar na solução do solo.

Tabela 3. Valores de umidade do solo (\%) nas posições de 5, 15, 25, $35 \mathrm{~cm}$ de distância do emissor e 15,30,60,90 cm de profundidade (média de nove coletas)

\begin{tabular}{|c|c|c|c|c|c|}
\hline \multirow{2}{*}{$\begin{array}{l}\text { Profundidade } \\
(\mathrm{cm})\end{array}$} & \multicolumn{4}{|c|}{ Distância Horizontal (cm) } & \multirow[b]{2}{*}{ Média } \\
\hline & 5 & 15 & 25 & 35 & \\
\hline 15 & $34 \mathrm{~A} / \mathrm{a}$ & $25 \mathrm{~B} / \mathrm{a}$ & $24 \mathrm{~B} / \mathrm{a}$ & $23 \mathrm{~B} / \mathrm{a}$ & $27 \mathbf{a}$ \\
\hline 30 & $27 \mathrm{~A} / \mathrm{b}$ & $27 \mathrm{~A} / \mathrm{a}$ & $25 \mathrm{~A} / \mathrm{a}$ & $24 \mathrm{~A} / \mathrm{a}$ & $26 \mathrm{a}$ \\
\hline 60 & $28 \mathrm{~A} / \mathrm{b}$ & $25 \mathrm{~A} / \mathrm{a}$ & $26 \mathrm{~A} / \mathrm{a}$ & $27 \mathrm{~A} / \mathrm{a}$ & $26 a$ \\
\hline 90 & $30 \mathrm{~A} / \mathrm{ab}$ & $28 \mathrm{~A} / \mathrm{a}$ & $27 \mathrm{AB} / \mathrm{a}$ & $23 \mathrm{~B} / \mathrm{a}$ & 27 a \\
\hline Média & 30 A & 26 B & $26 \mathrm{~B}$ & 24 B & \\
\hline $\begin{array}{c}\text { Teste F } \\
\text { Distância } \\
\text { C.V. }(\%)\end{array}$ & $\begin{array}{l}8,3 * * \\
16,0\end{array}$ & $\begin{array}{l}\text { Profundidade } \\
\text { DMS (média) }\end{array}$ & $\begin{array}{l}0,5^{\mathrm{ns}} \\
2,8^{2}\end{array}$ & $\begin{array}{l}\text { Dist. x Prof. } \\
\text { Média }\end{array}$ & $\begin{array}{l}2,6 * \\
26\end{array}$ \\
\hline
\end{tabular}

Letras iguais maiúsculas na horizontal e letras iguais minúsculas na vertical não diferem entre si pelo teste de Tukey a 5\% de probabilidade. Dist. = distância, Prof. = profundidade, C.V. = coeficiente de variação, DMS = diferença mínima significativa para a média. 
Tabela 4. Valores de pH na solução do solo, para as posições de 5, 15, 25, $35 \mathrm{~cm}$ de distância do emissor e 15, 30,60,90 cm de profundidade (média de nove coletas)

\begin{tabular}{|c|c|c|c|c|c|}
\hline \multirow{2}{*}{$\begin{array}{l}\text { Profundidade } \\
\text { (cm) }\end{array}$} & \multicolumn{4}{|c|}{ Distância Horizontal (cm) } & \multirow[b]{2}{*}{ Média } \\
\hline & 5 & 15 & 25 & 35 & \\
\hline 15 & $4,2 \mathrm{~B} / \mathrm{ab}$ & $4,0 \mathrm{~B} / \mathrm{a}$ & $5,1 \mathrm{~A} / \mathrm{a}$ & $4,1 \mathrm{~B} / \mathrm{b}$ & $4,4 \mathrm{a}$ \\
\hline 30 & $4,5 \mathrm{AB} / \mathrm{a}$ & $4,3 \mathrm{~B} / \mathrm{a}$ & $4,6 \mathrm{AB} / \mathrm{a}$ & $4,9 \mathrm{~A} / \mathrm{a}$ & $4,6 \mathrm{a}$ \\
\hline 60 & $4,1 \mathrm{~A} / \mathrm{ab}$ & $3,9 \mathrm{~A} / \mathrm{a}$ & $3,7 \mathrm{~A} / \mathrm{b}$ & $4,0 \mathrm{~A} / \mathrm{b}$ & $3,9 \mathrm{~b}$ \\
\hline 90 & $3,7 \mathrm{AB} / \mathrm{b}$ & $3,6 \mathrm{~B} / \mathrm{a}$ & $3,9 \mathrm{AB} / \mathrm{b}$ & $4,3 \mathrm{~A} / \mathrm{b}$ & 3,9 b \\
\hline Média & $4,1 \mathrm{AB}$ & $4,0 \mathrm{~B}$ & $4,3 \mathrm{~A}$ & 4,3 A & \\
\hline Teste F & & & & & \\
\hline Distância & $4,9 * *$ & Profundidade & $17,2 * *$ & Dist. $x$ Prof. & $4,3 * *$ \\
\hline C.V. (\%) & 8,4 & DMS (média) & 0,3 & Média & 4,2 \\
\hline
\end{tabular}

Letras iguais maiúsculas na horizontal e letras iguais minúsculas na vertical não diferem entre si pelo teste de Tukey a 5\% de probabilidade. Dist. = distância, Prof. = profundidade, C.V. = coeficiente de variação, DMS = diferença mínima significativa para a média.

Tabela 5. Valores de CE na solução do solo $\left(\mathrm{dS} \mathrm{m}^{-1}\right)$, nas posições de 5, 15, 25, $35 \mathrm{~cm}$ de distância do emissor e 15,30,60,90 cm de profundidade (média de nove coletas)

\begin{tabular}{|c|c|c|c|c|c|}
\hline \multirow{2}{*}{$\begin{array}{l}\text { Profundidade } \\
\text { (cm) }\end{array}$} & \multicolumn{4}{|c|}{ Distância Horizontal (cm) } & \multirow[b]{2}{*}{ Média } \\
\hline & 5 & 15 & 25 & 35 & \\
\hline 15 & $0,5 \mathrm{C} / \mathrm{c}$ & $0,7 \mathrm{~B} / \mathrm{b}$ & $0,9 \mathrm{~A} / \mathrm{b}$ & $0,8 \mathrm{AB} / \mathrm{b}$ & $0,7 \mathrm{c}$ \\
\hline 30 & $0,8 \mathrm{~B} / \mathrm{ab}$ & $0,9 \mathrm{AB} / \mathrm{a}$ & $1,0 \mathrm{~A} / \mathrm{b}$ & $0,4 \mathrm{C} / \mathrm{c}$ & $0,8 \mathrm{~b}$ \\
\hline 60 & $0,9 \mathrm{~B} / \mathrm{a}$ & $1,0 \mathrm{~B} / \mathrm{a}$ & $1,3 \mathrm{~A} / \mathrm{a}$ & $1,3 \mathrm{~A} / \mathrm{a}$ & 1,1 a \\
\hline 90 & $0,7 \mathrm{~A} / \mathrm{b}$ & $0,7 \mathrm{~A} / \mathrm{b}$ & $0,8 \mathrm{~A} / \mathrm{b}$ & $0,7 \mathrm{~A} / \mathrm{b}$ & 0,7 bc \\
\hline Média & $0,7 \mathrm{C}$ & $0,8 \mathrm{~B}$ & $1,0 \mathrm{~A}$ & $0,8 \mathrm{~B}$ & \\
\hline Teste F & & & & & \\
\hline Distância & $25,2 * *$ & Profundidade & $72,2 * *$ & Dist. x Prof. & $13,3 * *$ \\
\hline C.V. (\%) & 14,8 & DMS (média) & 0,09 & Média & 0,9 \\
\hline
\end{tabular}

Letras iguais maiúsculas na horizontal e letras iguais minúsculas na vertical não diferem entre si pelo teste de Tukey a 5\% de probabilidade. Dist. = distância, Prof. = profundidade, C.V. = coeficiente de variação, DMS = diferença mínima significativa para a média.

\subsection{Dinâmica do $\mathrm{NO}_{3}$ na solução do solo}

A concentração de $\mathrm{NO}_{3}$ na solução do solo logo abaixo do emissor foi baixa (419 $\mathrm{mg} \mathrm{L}^{-1}$ ), porém concentrações maiores do íon foram observadas a $35 \mathrm{~cm}$ de distância na horizontal e a $60 \mathrm{~cm}$ de profundidade $\left(849 \mathrm{mg} \mathrm{L}^{-1}\right)$, devido a sua alta mobilidade no solo, evidenciando que o seu caminhamento acompanha a frente de avanço da água até o extremo do bulbo (Tabela 6), neste ponto também foi observado o maior valor de CE (Tabela 5). Villas Bôas et al. (1999) observaram que o nitrato move-se para periferia da frente de molhamento e a concentração desse íon logo abaixo do emissor é pequena.

É interessante notar que mesmo a $35 \mathrm{~cm}$ de distância do emissor e a $90 \mathrm{~cm}$ de profundidade foi possível observar elevadas concentrações de nitrato, chegando a $624 \mathrm{mg} \mathrm{L}^{-1}$. Considerando a profundidade efetiva do sistema radicular da laranjeira de $60 \mathrm{~cm}$ (Pires et al., 2005), ocorreram perdas de $\mathrm{NO}_{3}$ em profundidade; isso justifica a importância da utilização de extratores de solução para o monitoramento de íons no solo. 
$\mathrm{Na}$ média, maiores concentrações de $\mathrm{NO}_{3}$ foram observadas nas profundidades de $30 \mathrm{e}$ $60 \mathrm{~cm}$ e a 25 e $35 \mathrm{~cm}$ de distância horizontal do emissor. Nesta situação ao considerar a instalação dos extratores de solução a $25 \mathrm{~cm}$ de distância do emissor e a $60 \mathrm{~cm}$ de profundidade é importante destacar o risco de perdas de $\mathrm{NO}_{3}$ por lixiviação, visto que esta localização está no limite da profundidade efetiva do sistema radicular da cultura. Destaca-se também o risco de não extrair solução nesta posição em condições de umidade baixa, por ser a periferia do bulbo.

Ao comparar à concentração de $\mathrm{NO}_{3}$ na posição $25 \mathrm{~cm}$ na horizontal e $60 \mathrm{~cm}$ na vertical com a posição $25 \mathrm{~cm}$ na horizontal e $30 \mathrm{~cm}$ na vertical, a variação de nitrato para as duas localizações foi de, aproximadamente, $15 \%$. Portanto, considerando o risco de lixiviação e a pequena variação entre os pontos mencionados anteriormente, recomenda-se instalar o extrator de solução para avaliação de nitrato a $25 \mathrm{~cm}$ de distância do emissor e a $30 \mathrm{~cm}$ de profundidade. Outro extrator a $25 \mathrm{~cm}$ de distância do emissor e a $60 \mathrm{~cm}$ de profundidade poderia ser instalado para monitorar perdas por lixiviação.

Tabela 6. Concentração de $\mathrm{NO}_{3}\left(\mathrm{mg} \mathrm{L}^{-1}\right)$ nas posições de $5,15,25,35 \mathrm{~cm}$ de distância do emissor e 15,30,60,90 cm de profundidade (média de nove coletas)

\begin{tabular}{|c|c|c|c|c|c|}
\hline \multirow{2}{*}{$\begin{array}{c}\text { Profundidade } \\
(\mathrm{cm})\end{array}$} & \multicolumn{4}{|c|}{ Distância Horizontal (cm) } & \multirow[b]{2}{*}{ Média } \\
\hline & 5 & 15 & 25 & 35 & \\
\hline 15 & $419 \mathrm{C} / \mathrm{b}$ & $531 \mathrm{~B} / \mathrm{ab}$ & $627 \mathrm{~A} / \mathrm{bc}$ & $574 \mathrm{AB} / \mathrm{bc}$ & $538 \mathrm{c}$ \\
\hline 30 & $481 \mathrm{C} / \mathrm{ab}$ & $598 \mathrm{~B} / \mathrm{a}$ & $770 \mathrm{~A} / \mathrm{a}$ & $500 \mathrm{C} / \mathrm{c}$ & 587 b \\
\hline 60 & $538 \mathrm{C} / \mathrm{a}$ & $482 \mathrm{C} / \mathrm{b}$ & $664 \mathrm{~B} / \mathrm{b}$ & $849 \mathrm{~A} / \mathrm{a}$ & $633 \mathbf{a}$ \\
\hline 90 & $451 \mathrm{C} / \mathrm{b}$ & $492 \mathrm{BC} / \mathrm{b}$ & $559 \mathrm{AB} / \mathrm{c}$ & $624 \mathrm{~A} / \mathrm{b}$ & 532 c \\
\hline Média & $472 \mathrm{C}$ & 525 B & $655 \mathrm{~A}$ & $636 \mathrm{~A}$ & \\
\hline $\begin{array}{c}\text { Teste F } \\
\text { Distância } \\
\text { C.V. (\%) }\end{array}$ & $\begin{array}{l}59,0 * * \\
8,9\end{array}$ & $\begin{array}{l}\text { Profundidade } \\
\text { DMS (média) }\end{array}$ & $\begin{array}{l}17,3 * * \\
43,0\end{array}$ & $\begin{array}{l}\text { Dist. x Prof. } \\
\text { Média }\end{array}$ & $\begin{array}{l}17,0 * * \\
573\end{array}$ \\
\hline
\end{tabular}

Letras iguais maiúsculas na horizontal e letras iguais minúsculas na vertical não diferem entre si pelo teste de Tukey a 5\% de probabilidade. Dist. $=$ distância, Prof. $=$ profundidade, $\mathrm{C} . \mathrm{V} .=$ coeficiente de variação, DMS = diferença mínima significativa para a média.

\subsection{Dinâmica do fósforo na solução do solo}

De acordo com a Tabela 7 a maior concentração de fósforo foi encontrada a $5 \mathrm{~cm}$ distancia horizontal do emissor e entre $15 \mathrm{~cm}$ e $30 \mathrm{~cm}$ de profundidade $\left(34 \mathrm{mg} \mathrm{L}^{-1}\right)$. Nota-se que a concentração deste nutriente é decrescente à medida que se afasta do ponto de emissão. A distribuição horizontal de fósforo na solução do solo limitou-se ao redor do gotejador, comportamento esperado devido a sua baixa mobilidade no solo. Na distância horizontal de 5 $\mathrm{cm}$ e na profundidade de $60 \mathrm{~cm}$ do emissor a concentração de fósforo diminuiu praticamente pela metade $\left(15 \mathrm{mg} \mathrm{L}^{-1}\right)$ em relação aos pontos de maiores concentrações $\left(34 \mathrm{mg} \mathrm{L}^{-1}\right)$.

O movimento do fósforo ocorre no solo quando são saturados os sítios de fixação, e como a água em solos arenosos apresenta movimento mais intenso em profundidade, formando bulbos estreitos na superfície e longos em profundidade (Tabela 3), maiores valores de fósforo foram observados em profundidade. Esse comportamento esta de acordo com Coelho (1994) e Souza (2006) que observaram movimentação de fósforo para camadas mais profundas do perfil do solo quando aplicado via técnica de fertirrigação por gotejo, em comparação com a adubação sólida convencional. 
Segundo Villas Bôas et al. (1999) a irrigação por gotejo pode aumentar o movimento de fósforo no solo de 5 a 10 vezes se comparado à aplicação sólida convencional. Laurindo et al. (2010) observaram que o sistema de gotejamento com uma linha de gotejadores por linha de plantas concentra no solo, sob o gotejador, maior quantidade de $\mathrm{P}$ aplicado via fertirrigação do que o de duas linhas, até a profundidade de $60 \mathrm{~cm}$. Lopes (1998) relata que o fósforo movimenta-se muito pouco no perfil do solo, permanecendo onde é colocado.

Deve-se considerar também que o fósforo já estava sendo aplicado na área a cerca de dois anos e possivelmente foi essa aplicação por longo período que promoveu o caminhamento em profundidade. Pode-se observar que mesmo a água chegando aos $35 \mathrm{~cm}$ de distância do emissor, o fósforo não acompanhou este caminhamento, pois a concentração de fósforo na solução do solo nos pontos mais distantes do emissor foi baixa (2 e $3 \mathrm{mg} \mathrm{L}^{-1}$ ).

Mesmo observando alto teor de fósforo na faixa de $5 \mathrm{~cm}$ de distância do emissor e a 15 e $30 \mathrm{~cm}$ de profundidade, deve-se ressaltar que próximo ao emissor coincide a região de maior teor de umidade no bulbo, denominada de zona saturada (Tabela 3). Sendo assim, nesta região podem ocorrer condições anaeróbicas, dificultando a absorção de nutrientes pelas plantas. Além disso, os maiores pontos de concentração de fósforo coincidiram com locais de baixos valores de $\mathrm{pH}$ (Tabela 4), mostrando que o ácido fosfórico foi um dos fertilizantes que maior contribuiu para a acidificação da solução. Considerando estas situações, recomenda-se a instalação dos extratores de solução a $15 \mathrm{~cm}$ de distância do emissor na horizontal e a $30 \mathrm{~cm}$ de profundidade.

Tabela 7. Concentração de fósforo $\left(\mathrm{mg} \mathrm{L}^{-1}\right)$, nas posições de $5,15,25,35 \mathrm{~cm}$ de distância do emissor e 15, 30,60, $90 \mathrm{~cm}$ de profundidade (média de nove coletas)

\begin{tabular}{|c|c|c|c|c|c|}
\hline \multirow{2}{*}{$\begin{array}{l}\text { Profundidade } \\
\text { (cm) }\end{array}$} & \multicolumn{4}{|c|}{ Distância Horizontal (cm) } & \multirow[b]{2}{*}{ Média } \\
\hline & 5 & 15 & 25 & 35 & \\
\hline 15 & $33 \mathrm{~A} / \mathrm{a}$ & $18 \mathrm{~B} / \mathrm{a}$ & $5 \mathrm{C} / \mathrm{a}$ & $4 \mathrm{C} / \mathrm{a}$ & $15 \mathbf{a}$ \\
\hline 30 & $34 \mathrm{~A} / \mathrm{a}$ & $12 \mathrm{~B} / \mathrm{ab}$ & $5 \mathrm{C} / \mathrm{a}$ & $5 \mathrm{C} / \mathrm{a}$ & 13 a \\
\hline 60 & $15 \mathrm{~A} / \mathrm{b}$ & $9 \mathrm{~B} / \mathrm{bc}$ & $3 \mathrm{C} / \mathrm{a}$ & $2 \mathrm{C} / \mathrm{a}$ & $7 \mathrm{~b}$ \\
\hline 90 & $3 \mathrm{~A} / \mathrm{c}$ & $3 \mathrm{~A} / \mathrm{c}$ & $2 \mathrm{~A} / \mathrm{a}$ & $2 \mathrm{~A} / \mathrm{a}$ & $3 \mathrm{c}$ \\
\hline Média & $21 \mathrm{~A}$ & $9 \mathrm{~B}$ & $4 \mathrm{C}$ & $3 \mathrm{C}$ & \\
\hline \multicolumn{6}{|l|}{ Teste F } \\
\hline Distância & $101,6 * *$ & Profundidade & $47,4 * *$ & Dist. $x$ Prof. & $15,7 * *$ \\
\hline C.V. $(\%)$ & 38,9 & DMS (média) & 3,1 & Média & 10 \\
\hline
\end{tabular}

Letras iguais maiúsculas na horizontal e letras iguais minúsculas na vertical não diferem entre si pelo teste de Tukey a 5\% de probabilidade. Dist. = distância, Prof. = profundidade, C.V. = coeficiente de variação, DMS = diferença mínima significativa para a média.

\subsection{Dinâmica do potássio na solução do solo}

As maiores concentrações de potássio foram observadas na faixa de $5 \mathrm{~cm}$ a $25 \mathrm{~cm}$ de distância do emissor na horizontal e entre 15 e $60 \mathrm{~cm}$ de profundidade (Tabela 8). Pode-se observar que a distribuição do íon ao longo do perfil foi homogênea, mostrando que o fluxo de $\mathrm{K}$ foi afetado pelo movimento da água no solo, pois a distribuição da umidade também foi bastante homogênea (Tabela 3).

Malavolta (2006) relata que o caminhamento do potássio junto com a água pode ser responsável por $25 \%$ da movimentação deste íon no solo. Uriu et al. (1980) comentam que a aplicação de potássio através da água de irrigação por gotejamento, promove distribuição uniforme do íon no bulbo úmido. Zanini (1991) observou maior concentração de K na região 
central do bulbo molhado, quando aplicado via fertirrigação por gotejo. Laurindo et al. (2010) observou que os teores de $\mathrm{K}$ no solo, quando aplicado via gotejamento em um pomar de laranjeiras, diminuíram radialmente com o aumento da distância horizontal do ponto de aplicação, na direção ortogonal à linha das plantas, até a profundidade de $60 \mathrm{~cm}$.

Devido à distribuição homogênea e considerando como ponto de referência a maior concentração de potássio no bulbo molhado $\left(115 \mathrm{mg} \mathrm{L}^{-1}\right)$, recomenda-se a instalação dos extratores de solução na faixa de $15 \mathrm{~cm}$ de distância do emissor e a $30 \mathrm{~cm}$ de profundidade.

Tabela 8. Média das concentrações de potássio $\left(\mathrm{mg} \mathrm{L}^{-1}\right)$, nas posições de $5,15,25,35 \mathrm{~cm}$ de distância do emissor e 15,30,60,90 cm de profundidade. (média de nove coletas)

\begin{tabular}{|c|c|c|c|c|c|}
\hline \multirow{2}{*}{$\begin{array}{l}\text { Profundidade } \\
\text { (cm) }\end{array}$} & \multicolumn{4}{|c|}{ Distância Horizontal (cm) } & \multirow[b]{2}{*}{ Méc } \\
\hline & 5 & 15 & 25 & 35 & \\
\hline 15 & $75 \mathrm{~A} / \mathrm{ab}$ & $88 \mathrm{~A} / \mathrm{a}$ & $70 \mathrm{~A} / \mathrm{b}$ & $77 \mathrm{~A} / \mathrm{a}$ & 77 \\
\hline 30 & $97 \mathrm{~A} / \mathrm{a}$ & $115 \mathrm{~A} / \mathrm{a}$ & $101 \mathrm{~A} / \mathrm{a}$ & $51 \mathrm{~B} / \mathrm{a}$ & 91 \\
\hline 60 & $96 \mathrm{~A} / \mathrm{a}$ & $91 \mathrm{~A} / \mathrm{a}$ & $75 \mathrm{AB} / \mathrm{ab}$ & $62 \mathrm{~B} / \mathrm{a}$ & 81 \\
\hline 90 & $54 \mathrm{~A} / \mathrm{b}$ & $57 \mathrm{~A} / \mathrm{b}$ & $57 \mathrm{~A} / \mathrm{b}$ & $53 \mathrm{~A} / \mathrm{a}$ & 55 \\
\hline Média & $80 \mathrm{~A}$ & $87 \mathrm{~A}$ & $76 \mathrm{~A}$ & $60 \mathrm{~B}$ & \\
\hline Teste F & & & & & \\
\hline Distância & $9,4 * *$ & Profundidade & $16,9 * *$ & Dist. $x$ Prof. & $3,4 * *$ \\
\hline C.V. $(\%)$ & 21,6 & DMS (média) & 13,8 & Média & 76 \\
\hline
\end{tabular}

Letras iguais maiúsculas na horizontal e letras iguais minúsculas na vertical não diferem entre si pelo teste de Tukey a 5\% de probabilidade. Dist. = distância, Prof. = profundidade, C.V. = coeficiente de variação, DMS = diferença mínima significativa para a média.

É importante ressaltar que este experimento foi realizado para cultura da laranja em apenas um tipo de solo (arenoso - comum na citricultura brasileira) e em condições específicas, como por exemplo, a vazão dos emissores $\left(2,3 \mathrm{~L} \mathrm{~h}^{-1}\right)$ e salientamos que há necessidade de estudos em diferentes solos e culturas para uma adequada recomendação de instalação dos extratores de solução de acordo com as condições de cultivo.

\section{CONCLUSÕES}

Baseado na mobilidade dos íons no perfil do solo recomenda-se instalar um extrator de solução do solo para determinar $\mathrm{P}$ e $\mathrm{K}$, localizado a $15 \mathrm{~cm}$ de distância do emissor na horizontal e a $30 \mathrm{~cm}$ de profundidade e para $\mathrm{NO}_{3}$ outro extrator localizado a $25 \mathrm{~cm}$ de distância do emissor na horizontal e a $30 \mathrm{~cm}$ de profundidade.

O extrator de solução mostrou ser uma ferramenta sensível, capaz de determinar a mobilidade dos íons no bulbo molhado.

\section{REFERÊNCIAS BIBLIOGRÁFICAS}

BLANCO, F, F. Manual de construção e utilização de extratores de cápsula porosa para obtenção da solução do solo. Teresina. Embrapa Meio-Norte, $1^{\text {a }}$ ed, 36 p, 2006. 
CAMARGO, O. A. Métodos de análise química, mineralógica e física de solos do Instituto Agronômico de Campinas. In: CAMARGO, O. A.; MONIZ, A. C; JORGE, J. A.; VALADARES, J. M. A. S. Campinas, Instituto Agronômico, 1986 (Boletim Técnico nº 106).

COELHO, A. M. Fertirrigação. IN: COSTA, E. F.; VIEIRA, R. F.; VIANA, P. A. Quimigação: aplicação de produtos químicos e biológicos via irrigação. Brasília: EmbrapaSPI, cap.8, p.201-227, 1994.

COELHO, R. B. Irrigação por gotejo: problemas e soluções. Citricultura Atual, Cordeirópolis, n.76, p.12-14, 2010.

FERREIRA, D. F. Sisvar versão 4.2. Lavras: DEX/UFLA, 2003.

LAURINDO, V. T. Interferência da fertirrigação, de lâminas e modos de aplicação localizada de água nos atributos químicos do solo e na produtividade da laranja Valência. 2005. 100f. Tese Dissertação (Mestrado em Produção Vegetal) - Faculdade de Ciências Agrárias e Veterinárias - Universidade Estadual Paulista, Jaboticabal. 2005.

LAURINDO, V. T.; SILVA, G. O.; PAVANI, L. C.; QUAGGIO, J. A. Padrão de distribuição de $\mathrm{K}, \mathrm{Ca}, \mathrm{Mg}$ e $\mathrm{P}$ no solo de um pomar de citros em função da fertirrigação. Engenharia Agrícola, Jaboticabal, v.30, n.5, p.909-921, 2010.

LOPES, C. C. Fertirrigacion: Aspectos básicos. IN: LOPES, C.C. Fertirrigacion: cultivos hortícolas y arnamentales. Madrid: Mundi-Prensa, 1998. p.63-80.

MAAS, E.V. Salinity and citriculture. Tree Physiology, Victoria, v.12, n.2, p.195-216, 1993.

MALAVOLTA, E. Manual de nutrição mineral de plantas. São Paulo: Editora Agronômica Ceres, 2006. 638p.

PIRES, C. R. M.; LUCHIARI, D. J. F.; ARRUDA, F. B. \& MOSSAK, I. Irrigação. In: MATTOS JUNIOR, D.; NEGRI., J. R.; PIO, R. M. \& POMPEU JUNIOR, J. Citros. Campinas, Instituto Agronômico e Fundag, p. 369 - 408, 2005.

QUAGGIO, J. A.; MATTOS JUNIOR, D.; CANTARELLA, H. Manejo da fertilidade do solo na citricultura. In: MATTOS JUNIOR, D.; DE NEGRI., J. D.; PIO, R. M.; POMPEU JUNIOR, J. Citros. Campinas: Instituto Agronômico e Fundag, 2005. p. 483 - 507.

RAIJ, B. Van.; ANDRADE, J. C.; CANTARELLA, H. \& QUAGGIO, J. A. Análise química para avaliação da fertilidade de solos tropicais. Campinas: Instituto Agronômico, 285p. 2001.

REICHARDT, K. A água em sistemas agrícolas. São Paulo: Manole, 1990. 188p.

SOUZA, T. R. Dinâmica de nutrientes no perfil do solo em sistemas de adubação sólida e fertirrigação na citricultura. 2006. 91f. (Dissertação de Mestrado)-Instituto Agronômico de Campinas. Campinas. 2006. 
URIU, K.; CARLSON, R. M.; HENDERSON, D. W. et al. Potassium fertilization of prune trees under drip irrigation. Jornal American Society for Horticultural Science, Alexandria, v.105, n.4, p.508-510, 1980.

VAN GENUCTHTEN, M. T. A. closed-from equation for predicting the hydraulic conductivity of unsaturated soil. Soil Science Society of America Journal, Madison, v.44, p.892-898, 1980.

VILLAS BÔAS, R. L.; BÜLL, L. T. \& FERNANDES, D. M. Fertilizantes em fertirrigação. In: FOLLEGATTI, M. V. (ed). Fertirrigação: citros, flores, hortaliças. Guaíba: Agropecuária, p. 293-319, 1999.

VILLAS BÔAS, R. L.; ZANINI, J. R.; DUENDAS, L. H. Uso e manejo de fertilizantes em fetirrigação. In: ZANINI, J. R.; VILLAS BOÂS, R. L.; FEITOSA FILHO, J. C. Uso e manejo da fertirrigação em hidroponia. Jaboticabal: FUNESP, 2002. p.1-25.

ZANINI, J. R. Distribuição de água e íon potássio no solo aplicados por fertirrigação em gotejamento. II. Teores de potássio no bulbo molhado. Irrigação e Tecnologia Moderna, Brasília, DF, v.46, p.24-38, 1991. 